\title{
AN IMPLANTABLE ALL-PARYLENE LIQUID-IMPEDANCE BASED MEMS FORCE SENSOR
}

\author{
Christian A. Gutierrez ${ }^{1}$, Connor McCarty ${ }^{1}$, Brian Kim ${ }^{2}$, Mrinal Pahwa ${ }^{3}$ and Ellis Meng ${ }^{1}$ \\ ${ }^{1}$ University of Southern California, Los Angeles, CA, USA \\ ${ }^{2}$ Duke University, Durham, NC, USA \\ ${ }^{3}$ University of Minnesota, Minneapolis, MN, USA
}

\begin{abstract}
We present a new transducer paradigm based on the electrochemical impedance transduction capability of encapsulated liquids within Parylene-based MEMS structures. We demonstrate the ability to measure forces in the micronewton range with a resolution of $4.56 \mathrm{~m} \Omega / \mu \mathrm{N}$. These sensors are ideally suited for applications requiring highly sensitive interrogation of soft non-planar surfaces in wet environments. Specifically, in situ and in vivo measurement of interfacial forces exerted on tissue by chronically implanted neural prosthetic devices is presently an unmet engineering challenge. Our approach enables, for the first time, interrogation of such biomechanical phenomena.
\end{abstract}

\section{INTRODUCTION}

Many force transduction methods are available (piezoresistive, capacitive, conductive polymers, optical, and ultrasound) spanning a wide variety of applications. However, often the substrate material and large sensor footprint preclude their use in the challenging wet environment of implantable-device/tissue interfaces. Quantification of parameters such as contact force and pressure is important to establishing their impact on device efficacy and functionality [1]. There is a need for highly sensitive sensors that can measure out-of-plane contact forces between soft-tissue and implanted devices at the local $\left(<500 \mu \mathrm{m}^{2}\right)$ level and have the ability to integrate with state-of-the-art polymer-based implantable devices such as an epi-retinal prosthesis [2].

To our knowledge, there is currently no sensing technology that adequately addresses this need. MEMS sensors based on electrochemical impedance transduction offer a novel approach with many advantages including low power consumption, small footprint, simplicity, and sensitivity. The advantages of electrochemically-based sensing have been extensively leveraged in applications such as cell counting [3, 4], but few attempts have been made to utilize this sensing modality in mechanical transducers. Emerging large-format impedance-based sensors have demonstrated detection of multiple parameters including point contact, force, direction, object shape, and texture [5]. Miniaturization of these sensors allows the targeting of $\mu \mathrm{N}-\mathrm{mN}$ force scales for detection of mechanical interactions between implanted prosthetics and tissue [6]. A novel Parylene-based contact force sensor for such applications is presented here.

\section{DESIGN AND OPERATION}

The sensor consists of a Parylene-C microchamber $(300 \mu \mathrm{m}$

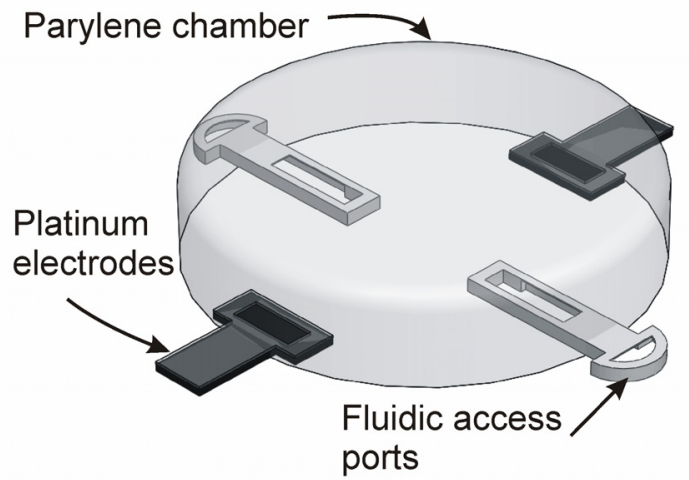

Figure 1 - All-Parylene force sensor layout and key

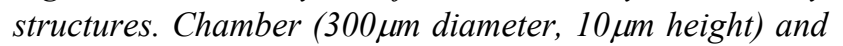
substrate are Parylene-C. Electrodes are Pt.

diameter) which provides a soft contact surface and a pair of thin film platinum microelectrodes (2000§ thick), exposed to the fluidic contents of the microchamber, that serve as an electrical interface for electrochemical measurement. Etched access ports located on the perimeter of the chamber connect the chamber to the external fluidic environment (Figure 1). Fluid is allowed to flow freely between the external environment and the chamber via the peripheral access ports.

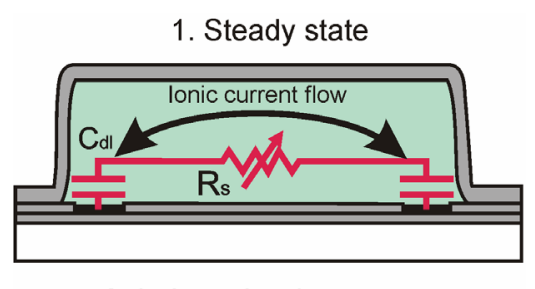

2. Indentation / contact / applied force

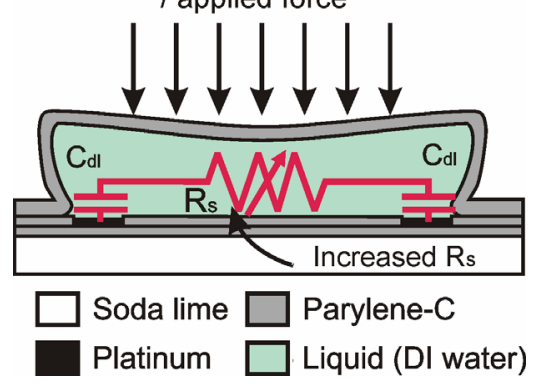

Figure 2 - Top: equivalent circuit model where $C_{d l}$ is the electrode double-layer capacitance and $R_{S}$ is the solution resistance. Bottom: under applied forces the crosssectional area between the electrodes decreases resulting in an increased $R_{S}$ and thus impedance magnitude.

During operation, external contact forces preferentially deform the compliant fluid-filled structure in the normal direction and redistribute the fluid contained within the 
chamber; some fluid may escape via the access ports. This deformation induces changes to the cross-sectional area available to current-carrying ions in the fluid and registers as a change in the magnitude of the solution impedance (Figure 2). Thus impedance variations can be correlated to mechanical contact forces exerted by external sources on the chamber diaphragm.

\section{THEORETICAL CONSIDERATIONS}

\section{Electrochemical impedance}

The equivalent circuit for impedance response can be simply approximated by the solution resistance, $R_{S}$, in series with the so-called double-layer electrode capacitance, $C_{d l}$. At sufficiently high frequencies $(>1 \mathrm{kHz})$, $C_{d l}$ is bypassed and $R_{S}$ dominates the impedance response. The impedance magnitude can then be modeled as a classical resistance Eq. (1) where impedance is inversely proportional to the cross-sectional area between the electrodes:

$$
|Z|_{f>1 k h z} \approx R_{S}=\frac{\rho l}{A} \approx \frac{\rho D}{2 \int_{0}^{a}(H-y(r)) d r}
$$

$\rho$ is the resistivity of the electrolyte, $D$ is the diameter of the chamber, $a$ is the chamber radius, $H$ is the chamber height, and $y(r)$ is the deflection of a circular membrane as a function of radius. Deflection, $y(r)$, through the axis of symmetry is used to approximate deflection through the entire radius of the device to simplify calculations.

\section{Fixed-edge diaphragm deflection approximation}

The diaphragm deflection $y(r)$ can be best described using a fixed-edge circular diaphragm model under small deflections. The deflection dependence relations under a uniformly distributed pressure and under a concentrated point force are given by Eq. $(2,3)$, respectively [7];

$$
\begin{aligned}
& y=\frac{3\left(1-\mu^{2}\right) P}{16 E t^{3}}\left(a^{2}-r^{2}\right)^{2} \\
& y=\frac{3\left(1-\mu^{2}\right)}{4 \pi} \frac{F}{E t^{3}}\left(2 r^{2} \ln \left(\frac{r}{a}\right)+a^{2}-r^{2}\right)
\end{aligned}
$$

where $y$ is deflection, $P$ is pressure, $F$ is force, $t$ is membrane thickness, $a$ is diaphragm radius, $E$ is modulus of elasticity, $r$ is radial distance, and $\mu$ is Poisson's ratio. By considering the deflection dependence under these two conditions, one can provide an upper and lower bound for the force-deflection sensitivity of a rigidly-clamped circular membrane under loads having differing contact areas. These bounds serve as useful design guides to evaluate device performance and capture key design variable relationships such as the cubic dependence on membrane thickness and quadratic dependence on device diameter.

\section{EXPERIMENTAL METHODS}

\section{Fabrication}

Standard surface micromachining techniques performed at low temperature $\left(90^{\circ} \mathrm{C}\right)$ were used for sensor fabrication (Figure 3). Briefly, Parylene-C was selected as the structural material for its combination of mechanical strength, electrical insulating properties, and


deposited and patterned on a Parylene coated $(10 \mu \mathrm{m}$ thick) soda lime wafer ( $1 \mathrm{~mm}$ thick) followed by a $1 \mu \mathrm{m}$ thick Parylene insulation layer. A $2 \mu \mathrm{m}$ sacrificial photoresist layer then formed the fluidic access ports followed by an additional Parylene deposition ( $2 \mu \mathrm{m}$ thick) and patterning step. A $10 \mu \mathrm{m}$ sacrificial photoresist layer was then spun-on and patterned establishing the chamber height and diameter $(300 \mu \mathrm{m})$ followed by deposition of a final $4.2 \mu \mathrm{m}$ thick Parylene film enclosing and forming the final chamber structure.



Figure 3 - Top: top view of device layout. Bottom: Low temperature $\left(90^{\circ} \mathrm{C}\right)$ process flow utilizing standard surface micromachining techniques. Cross-section taken through $B-B$ '.

The multilayer Parylene design provided electrical insulation to platinum traces, which may be routed through neighboring devices, thereby electrically isolating individual devices while reducing wiring complexity. Following removal of sacrificial photoresist by immersion in acetone and isopropyl alcohol, chambers were simultaneously filled at the wafer-level by passive soaking in a bath of the desired solution (DI water).

Device footprint was minimized by integrating fluidic access ports that allow fluidic access to the chamber while 
constraining the ionic conductive path within in the chamber interior environment. This enables the fabrication of dense sensor arrays (Figure 4) while minimizing the potential for crosstalk.

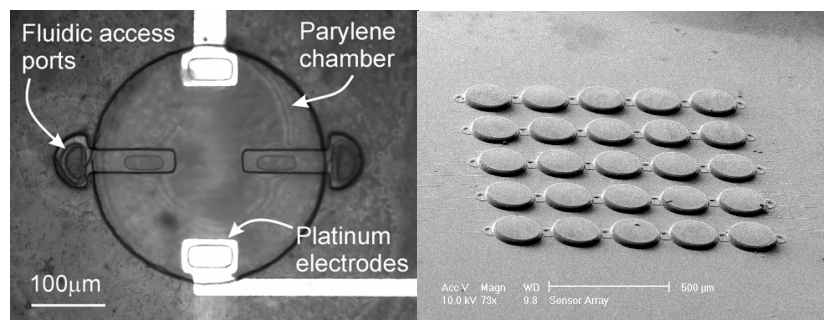

Figure 4 - Left: optical micrograph of a single fabricated device. Right: SEM of a 5x5 force sensor array demonstrating the dense integration capabilities of this technology. 25 sensors (200 um diameter) were fabricated within a 1mmx Imm footprint.

\section{Experimental setup}

Characterization experiments were conducted on a test die adhered and wire bonded to a printed circuit board. The device was maintained in a room temperature liquid environment and all measurements were performed while under complete immersion. The device characterization setup was built on a floating optical table and consisted of a motorized micropositioning stage with submicron accuracy to precisely control indentation depth and a Femtotools micronewton force probe for calibrated force measurements. Impedance was measured using a voltage divider circuit with a $5 \mathrm{kHz}, 1 \mathrm{~V}_{\mathrm{pp}}$ sinusoidal excitation signal and active rectification to attain the DC level of the AC response. The circuit response was calibrated using an Agilent E4980A precision LCR meter. A Keithley 2400 precision multimeter was used to record the real-time impedance response. The motorized stage, force probe, and multimeter were all interfaced through a custom LabVIEW interface for real-time data acquisition.

\section{RESULTS AND DISCUSSION}

Characterization of the force-deflection response was measured by indenting the center of the chamber with the Femtotools force probe mounted onto the micropositioning stage. The force probe effector tip consisted of silicon shank terminating in a $50 \mu \mathrm{m} \times 50 \mu \mathrm{m}$ flat face. Devices of $300 \mu \mathrm{m}$ diameter and $10 \mu \mathrm{m}$ height were indented in $1 \mu \mathrm{m}$ steps while immersed in DI water and exhibited a linear force-deflection response (Figure 5). DI water was selected because of the inherently high resistivity, ensuring that solution resistance dominated the sensor impedance response.

The measured force curve falls within the response bounds predicted by the theoretical model in agreement with the indentation of the chamber using a contact area smaller than the overall device area as is the case previously noted for the force probe. The linear response obtained also agrees well with the force-deflection response predicted by theory. This observation also indicates that the presence of liquid in the chamber has a limited effect on the overall mechanical response of the system. The use of a higher viscosity medium in the chamber would likely alter this condition by increasing the slope of the force-deflection curve. The response can also tuned by adjusting parameters such as device diameter, membrane thickness, and membrane composition.

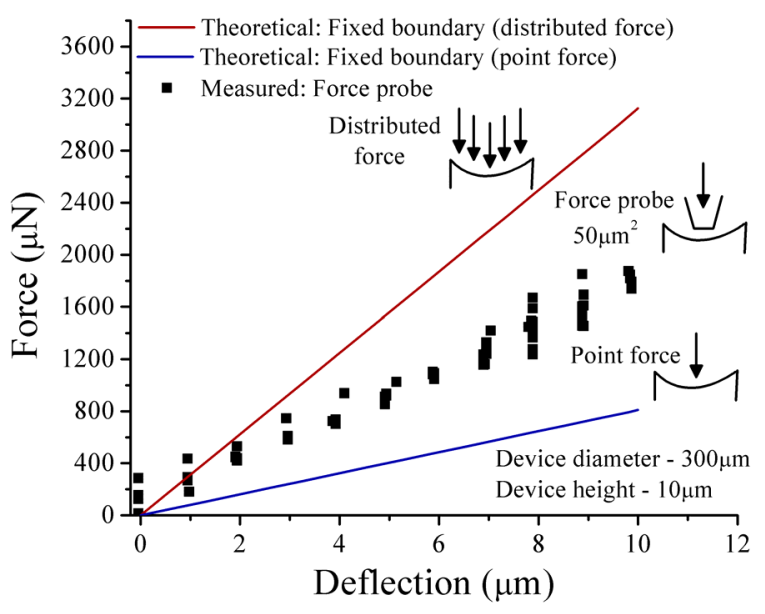

Figure 5 - Force-deflection response. Theoretical responses for a rigidly clamped circular membrane under distributed and concentrated point force conditions bound the measured response. Force probe contact area is $50 \mu m \times 50 \mu m$. Small deflection model accurately captures linear response.

A linear relationship between deflection and chamber cross-sectional area can also be predicted by Eq. (1). This relation is valid throughout a majority of the deflection range but degrades near full deflection, at which point, the membrane touches down to the substrate. The inverse relationship between impedance and area therefore predicts a characteristic non-linear inversely proportional impedance-deflection relationship. The measured data agree well with this prediction within about $80 \%$ of full deflection but the measured impedance asymptotically reaches a maximum value as maximum deflection is reached and the membrane touches down (Figure 6).

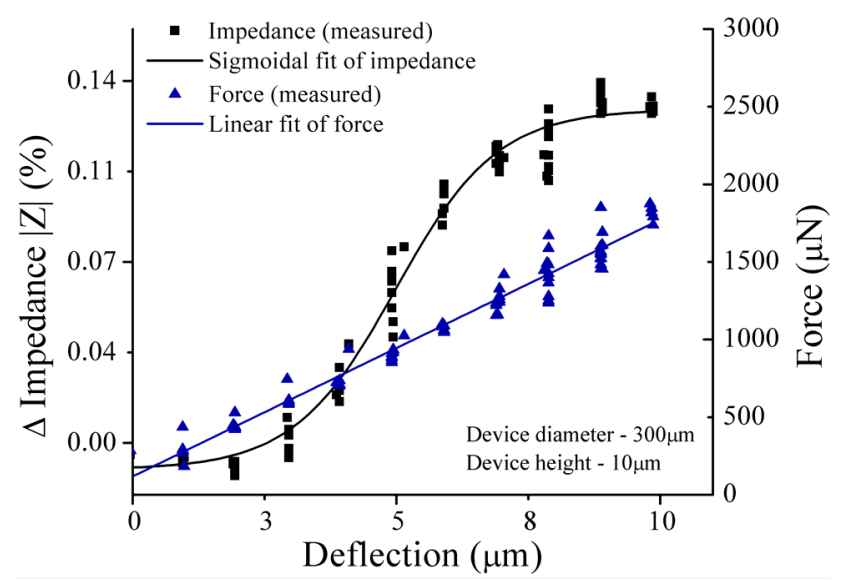

Figure 6 - Impedance and force response vs deflection depth. Force-deflection follows a linear trend while impedance-deflection shows a non-linear dependence. The impedance is function of the ionic conductive path between the electrodes which is governed by the change in cross-sectional area available for ionic current flux. 
The measured impedance-deflection response is closely fitted by a sigmoidal curve over the full range of deflection. Although this relationship is clearly evidenced by the measured response, full membrane deflection should be avoided in order to prevent any damage to the device. Maximum deflection should therefore be limited to within $80 \%$ of maximum device height or the range over which the $|Z| \propto 1 / A$ proportionality holds.

For the intended application, as an impedance-based force sensor, the impedance-force relationship needs to be considered. The measured response (Figure 7) clearly shows a linear region of operation between $500-1500 \mu \mathrm{N}$ yielding an impedance-force measurement resolution of $1.4 \mathrm{ppm} / \mu \mathrm{N}(4.56 \mathrm{~m} \Omega / \mu \mathrm{N})$. In addition to device geometry, this response can be tuned by adjusting the solution resistivity, $\rho$. The data shown here were measured in DI water but further experiments are planned in more conductive electrolytes such as saline that mimic the body's natural environment.

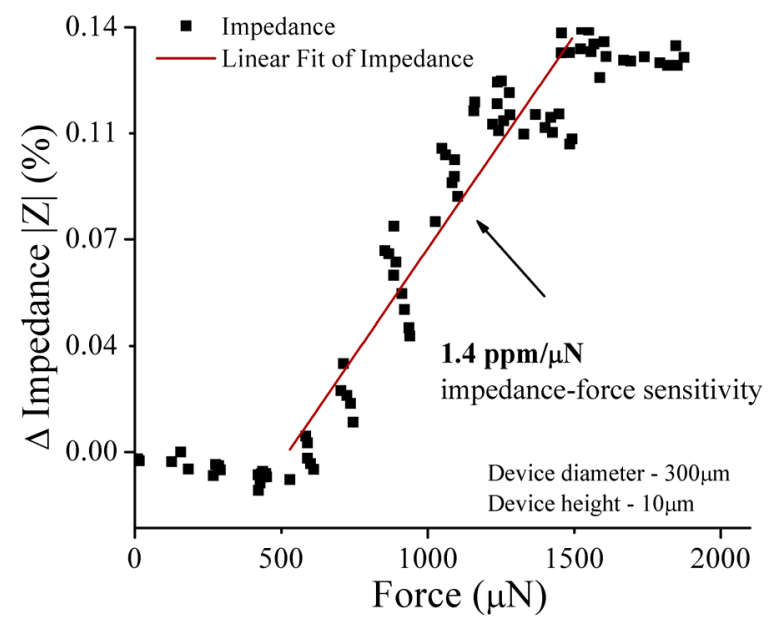

Figure 7 - Impedance response to force acting on chamber membrane. Force probe provides a $50 \times 50 \mathrm{~mm}$ contact area. Device sensitivity of $1.4 \mathrm{ppm} / \mu \mathrm{N}$ $(4.56 \mathrm{~m} \Omega / \mu N)$ measured in linear region of operation.

The ability to measure forces on the $\mu \mathrm{N}-\mathrm{mN}$ force scales using a polymer-based structure enables these sensors to be integrated with existing polymer-based implantable technologies. By utilizing the surrounding liquid environment as the sensing medium rather than seeking to hermetically seal or isolate the sensor from the medium greatly facilitates packaging. This advantage comes at the cost of added calibration requirements to ensure accurate measurement upon application in the intended medium. The addition of self-sealing structures, such as an integrated stiction valve, adds long-term liquid encapsulation capability enabling use in dry applications $[6,8]$. Additionally, the ability to fabricate these devices completely out of Parylene-C enables the release from rigid substrates and provides the ability to place these sensors on non-planar surfaces and in other locations and environments which have been traditionally very difficult to access, such as device-tissue interfaces. Contact force experiments utilizing ex vivo retinal tissue samples are planned to validate sensor operation with soft tissue.

\section{CONCLUSION}

We designed, fabricated and tested an all-Parylene electrochemical impedance-based contact force sensor. A classical circular diaphragm deflection model accurately predicted the range of operation for the device under different loading conditions. We also demonstrated the ability to measure forces in the micronewton range with a resolution of $4.56 \mathrm{~m} \Omega / \mu \mathrm{N}$. These sensors are ideally suited for applications requiring highly sensitive contact force measurements of soft non-planar surfaces in wet environments and allow for the first time interrogation of such mechanical phenomena.

\section{ACKNOWLEDGEMENTS}

This work was funded in part by the Engineering Research Centers Program of the NSF under Award Number EEC-0310723 and the Bill and Melinda Gates Foundation (CG). The authors would like to thank Dr. Donghai Zhu and the members of the USC Biomedical Microsystems Laboratory for their assistance.

\section{REFERENCES}

[1] J. D. Weiland, W. Liu, et al., "Retinal Prosthesis," Annual Review of Biomedical Engineering, vol. 7, pp. 361-401, 2005.

[2] D. C. Rodger, A. J. Fong, et al., "Flexible parylene-based multielectrode array technology for high-density neural stimulation and recording," Sensors and Actuators B: Chemical, vol. 132, pp. 449-460, 2008.

[3] H. P. Schwan, "Electrode polarization impedance and measurements in biological materials," Ann. New York Acad. Sci., pp. 191-209, 1967.

[4] O. F. Schanne and E. R. P.-. Ceretti, Impedance measurements in Biological Cells: John Wiley \& Sons, 1978.

[5] N. Wettels, V. J. Santos, et al., "Biomimetic Tactile Sensor Array," Advanced Robotics, vol. 22, pp. 829-849, 2008.

[6] C. A. Gutierrez and E. Meng, "A Dual Function Parylene-based Biomimetic Tactile Sensor and Actuator for Next Generation Mechanically Responsive Microelectrode Arrays," in Proc. Transducers 2009, Denver, CO, USA, pp. 21942197.

[7] M. D. Giovanni, Flat and Corrugated Diaphragm Design Handbook, vol. 11. New York, New York: Marcel Dekker Inc, 1982.

[8] Z. Wang and Y. Xu, "Theoretical and Experimental Study of Annular-Plate SelfSealing Structures," Microelectromechanical Systems, Journal of, vol. 17, pp. 185-192, 2008. 